\title{
Research on Control System for Whole Process of Informatization
}

\author{
Xiaoyi Yu ${ }^{1, \text { a }}$, Yan Cui ${ }^{2}$ \\ ${ }^{1}$ China Southern Grid Co., Ltd, Guangzhou 510623, China \\ ${ }^{2}$ GuangDong Electric Power Design Institute, Guangzhou 510663, China \\ ayuxiaoyi989@163.com
}

Keywords: Control System, Enterprise Architecture.

\begin{abstract}
The traditional IT Management and control system has several problems. Firstly, focus on many points such as designing, projects and documents. The whole process is not connected and controlled by the system. Secondly, the lack of flexible results in the inconsistent of control process and application building process. Thirdly, many works are carried out manually, and is lake of efficiency. In this paper, we renewed the traditional IT Management and control system from 4 aspects (mechanism, method, object, and platform) and built a new system that can control the whole process of informatization, gathering and interrelating all the control objects, using the automatic tools to carry out the management and control work more efficiently.
\end{abstract}

\section{Introduction}

Information control is the cornerstone of information construction. Relying on information control, enterprises can overall control all types of IT resources and their relationships. It can follow the overall strategy, supported by enterprise architecture and landing effectively, by effective integration with business architecture to ensure information technology can effectively support the business.

Currently, many companies (especially large enterprises) established their own information management and control system. Information management and control system has also become a hot academic research. FengYuhas construct a complete enterprise information management and control system [1] based on 5 factors which are culture, strategy, organization, operation, and monitor. Jie Hu and others has proposed the multinational information technology overall strategy and deployment models based on internationalization features of four stages.

However, there are inherent problems in traditional system of information control which is unable to meet the needs of information control and management under new context, as follows:

1) In terms of control mechanisms, management and control process has not run through from design, development, implementation to operation, there is lacking of methods and rules to ensure the consistency from design, development, implementation and operation.

2) For control methods, under the guidance of traditional top-down enterprise architecture methodology which lack of the ability to quickly adjust the architecture design according to the changing business. The Flexibility of the traditional control system cannot meet the needs of enterprise business innovation and development.

3) In terms of control objects, take the design document as a carrier, and lack of effective control of development, deployment, implementation and operation scheduling. The integrity of the traditional control system cannot adapt to the requirement which are information integration, outcome knowledgeable and the quality can be monitored of enterprise information management and control information.

4) There is a lacking of a run through the whole process, automated management and control platform. The operation efficiency of the conventional control system cannot meet the needs of large-scale enterprises to carry out dynamic enterprise members to management and control work informational. 


\section{Connotation of whole process oriented information management control}

Information management and control is the scientific description of various types of information elements (IT resources) and its relationship to establish a reasonable compliance, consistent, dynamic preservation of IT resources system. And relying on the control method and control tools to achieve information technology construction from the demand, design, development, implementation, deployment and operation of the whole process and automated control. The overall framework of information management and control shown as below:

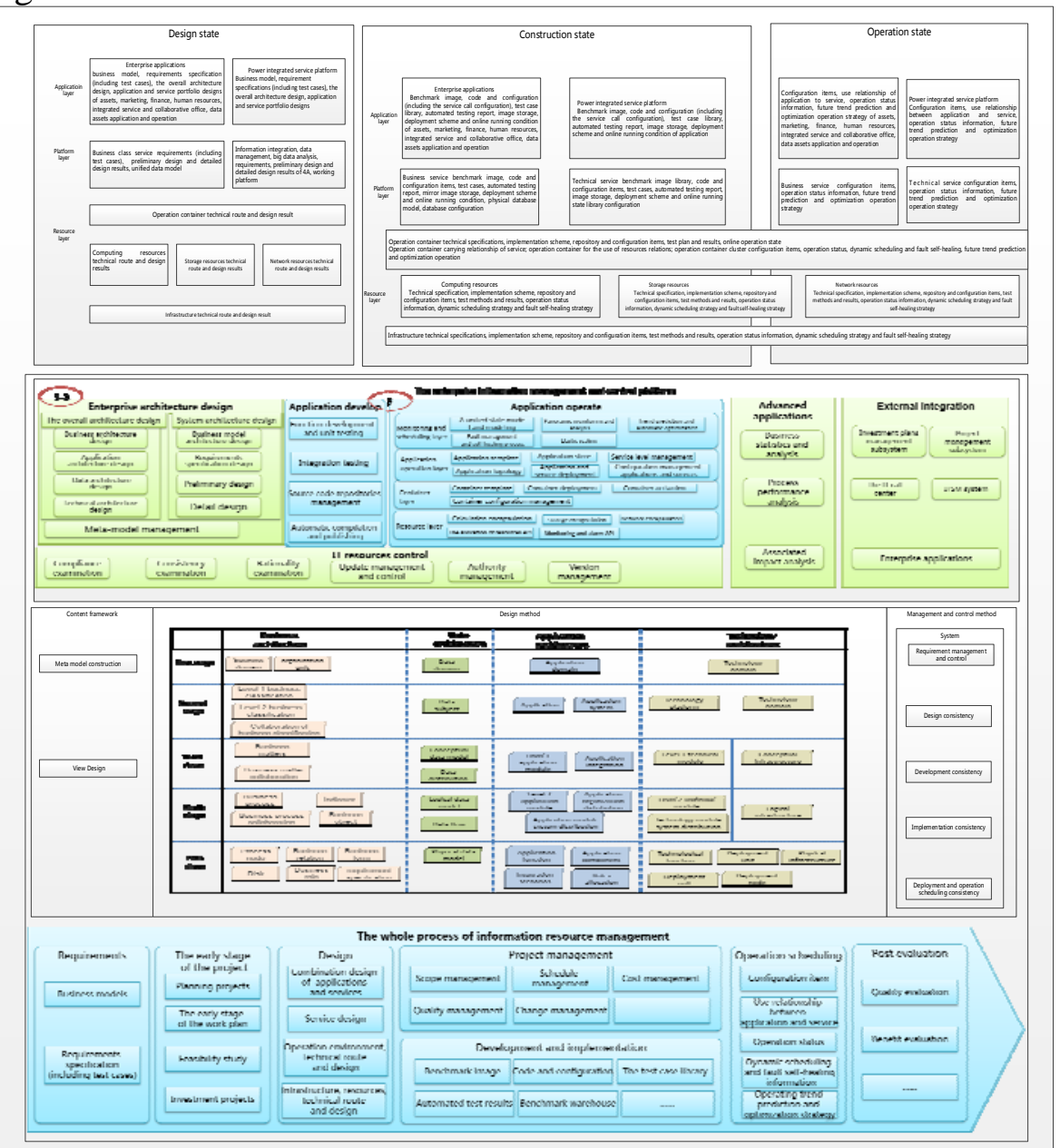

To management and control the whole process of information construction, the core is to put forward EA to the front-end, run through the management and control mechanism, method, object (IT resources) in the information process of demand, design, development, implementation, deployment and operation.

The whole process of information management control mechanism:

The whole process of information management and control as the main line, establishing a management control system and mechanism that covers demands, project pre-stage, design, development, implementation, operation scheduling and post-evaluation stages, also establishing organizations and teams adapting to the whole process of information management and control to achieve enterprise information of the control system and mechanism of efficient and sustainable operation.

1) Demand: to achieve business needs based on the unified modeling and unified control of overall blueprint of the enterprise architecture (mainly business model), and fully ensure the rationality, compliance and consistency with the overall blueprint of the demand. Reasonable business requirements will form the standard requirements specification (including test cases).

2) The project early stage: collect the relevant requirements specifications for the project, and effective control the feasibility and investment estimates of the project. 
3) Design: According to the overall blueprint of enterprise architecture and service-oriented IT architecture, carry out the management and control of application and service combination design and service design, control the business needs of the implementation methods and details.

4) Development and implementation: on the one hand, apply the project management, supervision and other related methods comprehensively, to carry out project construction control in project scope, schedule, cost and quality; On the other hand, manage and control the operation environment, code, configuration items, test cases and test results of applications and services.

5) The operation of scheduling: Based on standardized operating environment to run the operation. In the process of dispatching, overall control the relationship and operate status of applications, services, operation environment and basic resources, and carry out the dynamic scheduling, fault self-healing and trend forecast to improve the forward-looking, pertinence and automation level and efficiency of operation scheduling.

6) Post-evaluation: Manage and control the post-project evaluation, control the quality and effectiveness of the project, form the closed-loop feedback mechanism to promote the overall process of continuous optimization and improvement.

\section{Whole process oriented information control method}

Information design methods for the whole process and control method takes the object-oriented architecture design and integration of business information as a guide. By absorbing the company's past enterprise architecture experience, establish the improved IT resources framework (including meta-model and view design), design method (including all types of elements and their relationships definition, identification and design methods, etc.) and control methods (including control standards, norms and rules, etc.) system, and form the relevant standards.

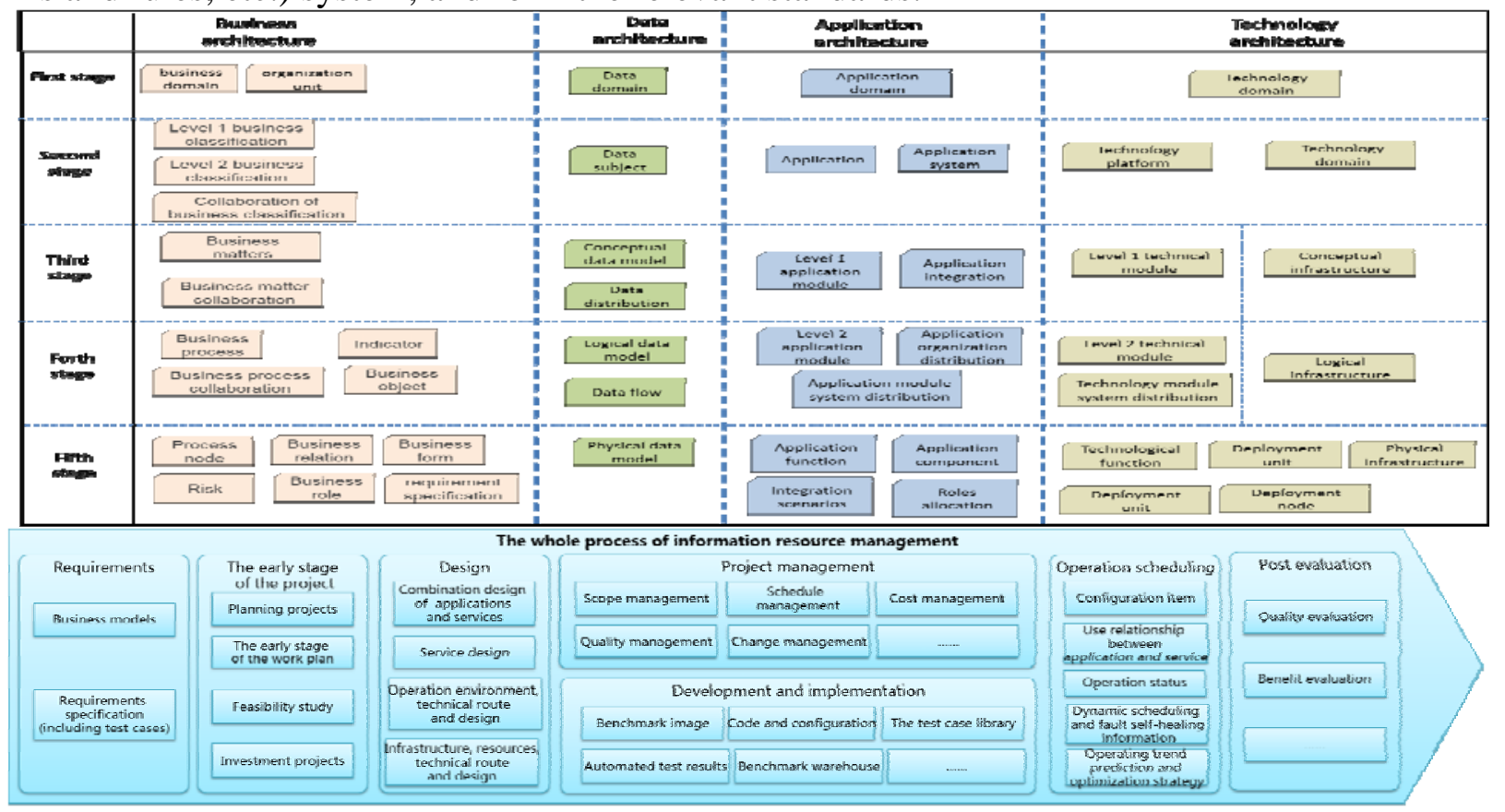

\section{Whole process oriented information management and control platform}

Information management and control for the whole process run through the management and control platform of modeling, design, control, operation scheduling for management as the supporting tools. By using the enterprise architecture design and management, agile development, development and operation (DEVOPS), cloud operation scheduling means or tools. 


\section{Whole process oriented IT resources system}

Whole process oriented informatization control is taking the systematic, strong consistency IT resources system as the carrier. Fully inherited the company existing enterprise architecture design, including design, construction and operation status, etc., covering business, application, data and technical types, connection between each element, strong consistency of IT resources system.

1) In the design state, unified summarize, refine, structure and associate the application layer, platform layer and resource layer designs. In the application layer, management results including business models, requirement specifications and overall architecture design and system architecture design, etc. Among them, the requirements specification will be refined to the test case, as the main basis of control in construction state to carry out the development achievements. Relying on the related mechanism, to keep the application design dynamic preserved; system architecture design results covers application system layer, control layer. And the call combine relationship between application and service portfolio.

2) In the construction state, based on standardized operating environment that is running through the test and operation based on the running containers. On this basics, applications was packaged according to the standard mirror images, manage the operation environment of applications and services(Benchmark image), code and configuration items (including service invocation configuration), test case library, automated test report, mirror repository, deployment and online status, to form a unified configuration database across applications, services, running containers, resource pools and infrastructure to support the unification of development, operation and maintenance, and dynamic operation scheduling mode.

3) In the running state, according to the operating environment and configuration library, manage the operation status, dynamic scheduling strategy, fault self-recovery strategy, trend analysis and optimization strategies of application layer, platform layer and resource layer to unify operation and management, operation and maintenance, operation and monitor of application, service and resource, supporting auto deployment, dynamic monitor, fault positioning, fault self-healing, service level management, trend forecasting and optimization of application, service and resource, to effectively enhance the perceptiveness, pertinence and automation level and efficiency.

\section{Conclusion}

In this paper, the whole process of informatization construction can be traced, flexible and adaptable, with comprehensive results and consistent, and automatic control the work automation of total new information management and control system, which there is great significance for the whole process of information management and control.

However, there are still lots of contents in the whole process oriented information management and control system need to deepen and improve, especially in the automated control tool system and platform construction and integration, need to achieve deep integration of cloud computing, DevOps and other technical means and traditional information management tools. Only under the support of automated control tool system and platform, the whole process oriented information management and control can be effectively carried out. This requires the joint efforts of researchers, developer and other stakeholders.

\section{References}

[1] Feng Yu, The construction of enterprise information control system, Command information system and technology, 2012, 3(2):38-43.

[2] HaoHu, Hao-tong Zhao, GangWang, Informationization strategy research under the control mode of Multinational enterprise, Chinese management informatization, 2012, 15(9):75-77.

[3] WenliYou, TongWang, Thoughts of the present situation of the enterprise information management in our country, Business research, 2003(2):24-26. 